\title{
Interprofessional ECMO telerounding: a novel approach to neonatal ECMO clinical participation and education
}

\author{
Brianna K. Brei $\mathbb{( i )}^{1} \cdot$ Megan M. Gray $\mathbb{( i D}^{1} \cdot$ Rachel Umoren ${ }^{1}{ }^{1} \cdot$ Sarah Handley ${ }^{1} \cdot$ Robert DiGeronimo ${ }^{1}$. \\ Taylor Sawyer $\mathbb{1}^{1} \cdot$ Kendra Smith $\mathbb{B}^{1} \cdot$ Zeenia Billimoria $^{1}$
}

Received: 15 June 2020 / Revised: 21 August 2020 / Accepted: 14 September 2020 / Published online: 22 September 2020

(C) The Author(s), under exclusive licence to Springer Nature America, Inc. 2020

\begin{abstract}
Objective Determine the feasibility, strengths, and barriers of offering extracorporeal membrane oxygenation (ECMO) telerounding to neonatal intensive care unit (NICU) care providers.

Study design NICU providers were invited to join ECMO rounds by teleconference. Data were collected on telerounding participation and ECMO concepts discussed. A survey was sent to all providers.

Results From March 2018 to February 2020, telerounding on 24 neonatal ECMO patients (168 ECMO days) was performed in a Level IV NICU. A mean of four providers joined telerounds per ECMO day with an increase from 3 to 6 providers over the study period. Nearly all respondents felt telerounding lowered barriers to attending ECMO rounds (94\%), promoted engagement (89\%), and improved continuity of care (78\%). Barriers to ECMO telerounding were suboptimal audio connections and limited ability to participate in the clinical discussion.

Conclusion ECMO telerounding is well-received by NICU providers. It can improve provider participation, complement existing in-person ECMO rounds, and ECMO education.
\end{abstract}

\section{Introduction}

Extracorporeal membrane oxygenation (ECMO) provides life support to neonatal patients with cardiorespiratory failure refractory to conventional therapy. Neonatal ECMO is a high acuity, yet low occurrence event. ECMO utilization in neonates is increasing [1], however, ECMO for neonatal respiratory failure is decreasing due to advancements in medical management [2,3]. Many pediatric ECMO centers have less than 15 pediatric patients a year of which $\sim 60 \%$ are neonates [4]. This means many programs will have limited opportunities for clinical participation and experiential learning of the intricacies of neonatal ECMO.

Supplementary information The online version of this article (https:// doi.org/10.1038/s41372-020-00827-4) contains supplementary material, which is available to authorized users.

Zeenia Billimoria

zeenia.billimoria@seattlechildrens.org

1 Department of Pediatrics, Division of Neonatology, University of Washington School of Medicine and Seattle Children's Hospital, Seattle, WA, USA
The Extracorporeal Life Support Organization has specific educational guidelines on the training of ECMO specialists. This training involves a combination of didactics, water drills, simulation, animal labs, and bedside training [5]. However, there are no specific certification or recertification requirements for physicians and other healthcare practitioners directing care for these critically-ill patients [6]. There is significant variability among pediatric ECMO centers in ECMO skills acquisition and maintenance. Even for the most experienced providers, knowledge and skills decrease over time in the absence of routine clinical exposure [7]. Given the complexity and infrequent occurrence of neonatal ECMO cases, providers who care for these patients may need to obtain more experience through the use of multiple platforms [8], including virtual or low fidelity means.

Telemedicine has been used in neonatal care since 1998 when the technology was first used in North Queensland, Australia to unite babies in the neonatal intensive care unit (NICU) with their mothers and families across long distances [9]. Recently, many studies have shown the benefit of telemedicine in regards to neonatal resuscitation care [10], rounds [11, 12], simulation education [13], and NICU follow-up care [14]. In pediatric ECMO care, telemedicine 
has been used for provider to provider consultation [15], but these reports have not focused on provider clinical participation in the care of ECMO patients.

We explored the feasibility of neonatal ECMO telerounding to increase opportunities for healthcare providers at our institution to participate in the care of neonatal ECMO patients. We hypothesized that ECMO telerounding would be feasible to implement, would improve provider participation in ECMO cases, and complement existing inperson ECMO rounds.

\section{Methods}

\section{Study design}

From March 2018 to February 2020, we conducted a single center prospective observational study of all neonatal providers participating in ECMO rounds at a Level IV NICU. Neonatologists, neonatal fellows, and neonatal advanced practice providers who were part of the division of neonatology were invited to join ECMO rounds in-person or by telerounds remotely. Prior to this study, all neonatal providers were informed of a new ECMO patient on day 1 of ECMO and were invited to participate in daily in-person rounds. Data on telerounding participation were collected prospectively. A survey was used to examine perceived strengths and barriers to ECMO telerounding. This study was approved and deemed exempt by the Seattle Children's Institutional Review Board PIROSTUDY15919.

\section{Telerounding procedures}

In-person ECMO rounds were teleconferenced in real time daily during the study period. Rounds typically occurred between 8:10 and 8:45 a.m. Telerounds were held using Zoom (Zoom Video Communications, Inc., San Jose, CA, USA), a HIPAA compliant teleconferencing software application. During rounds, an iPad (Apple Inc., Cupertino, CA, USA) with Zoom installed was set up on a beside stand to broadcast rounds. An eMeet M1 wireless Bluetooth conference speaker (Shenzhen eMeet Technology Co., Ltd. Shenzhen, Guangdong, China) was used to capture telerounding audio. Only audio, not video, was transmitted as it was challenging to capture the entire in-person team. One neonatologist was tasked to moderate telerounds each day, and to ensure the telerounding software and speaker were connected and working. The moderating neonatologist was separate from the rounding neonatologist and ECMO neonatologist when possible.

Prior to rounds, the pediatric resident and neonatal fellow completed an ECMO rounding sheet. To ensure that remote participants had pertinent patient data without accessing the electronic medical record, the moderating neonatologist sent via secure email the clinical data on the ECMO patient(s) to a NICU provider listserv consisting of all neonatologists, neonatal fellows, and neonatal advanced practice providers prior to ECMO rounds. The email contained a copy of the ECMO rounding sheet which included a one-line patient synopsis, daily lab results, ventilatory support, medications, and other ECMO related information as well as the most recent radiograph. The email also contained the Zoom meeting details with options to connect by Zoom app, weblink, or by phone.

In-person participation on ECMO rounds primarily included 2 neonatal rounding teams (Team 1: one neonatologist, one neonatal fellow, three pediatric residents; Team 2: one neonatologist, two advanced practice providers), a dedicated ECMO neonatologist, a nutritionist, a pharmacist, the bedside nurse, the respiratory therapist, the ECMO specialist, and occasionally non-clinical neonatologists and/or neonatal fellows. Depending upon the case, pediatric surgeons, surgery fellows, and nephrologists would attend in-person for the multidisciplinary ECMO rounds. All remote participants were muted but could ask questions using the Zoom "chat" function. The in-person moderating neonatologist would read pertinent comments or questions to the in-person rounding team. Responses were then provided by the in-person team or by the moderating neonatologist.

Due to early feedback about audio quality during telerounds, several interventions were made to improve the sound environment during the study. These included (1) reminding the in-person team presenting on rounds of remote participants listening in, (2) placing the Bluetooth conference speaker on a rolling cart in the middle of the inperson rounding team and moving it closer to the team member speaking, and (3) silencing patient alarms in a timely fashion during rounds.

\section{ECMO telerounds data collection}

Data on the number of remote participants, in-person participants, provider type, type of ECMO, ECMO concepts discussed, and any teleconferencing issues encountered during rounds was collected daily by the moderating neonatologist. This data was maintained in a secure database.

\section{Telerounding survey development and distribution}

A survey was developed by the investigators [ZB, MG] to evaluate the experience of ECMO telerounds. Survey questions are shown in Supplementary Table 1. The survey was administered in December 2018 to all neonatology providers in the division via a Research Electronic Data Capture (REDCap) link sent by email. By this time period, 


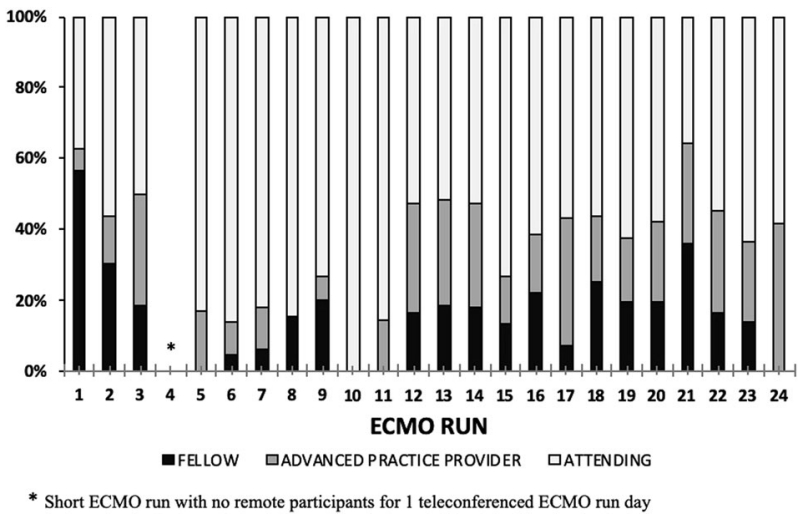

Fig. 1 Remote participant distribution by role.

ten ECMO runs had been conducted by telerounds allowing for multiple opportunities for a variety of neonatal providers to have participated remotely by telerounds. Only those providers who had attended ECMO rounds remotely were asked to take the survey. These same providers may also have attended ECMO rounds in-person. We identified 29 neonatology providers who had participated in ECMO rounds remotely prior to December 2018 based on survey response and data gathered on remote participants. The survey was open for 1 week and all responses were collected anonymously.

\section{Statistical analysis}

Data were analyzed using descriptive statistics. Categorical data are presented as counts $(n)$ and percentage (\%). Statistical analysis was performed using Microsoft Excel (Microsoft Inc., Redmond, WA, USA). Because the data were descriptive, no tests of statistical significance were performed. Data on 24 ECMO rounding days were excluded from the final analysis due to incomplete participant data, resulting in a total of 168 ECMO days for analysis.

\section{Results}

During the study period, telerounds were performed on 24 neonatal ECMO patients totaling 168 ECMO run days. On average, four neonatal providers (two neonatologists, one neonatal fellow, one advanced practice provider) participated remotely in telerounds per ECMO run day (Fig. 1). The average number of remote participants increased from 3 to 6 per ECMO run day during the study (Fig. 2).

The survey response rate was $62 \%$ (18), of which $17 \%$ (3) were fellows, $6 \%$ (1) were advanced practice providers, and $78 \%$ (14) were neonatologists. Remote telerounding participant level of ECMO and teleconferencing experience

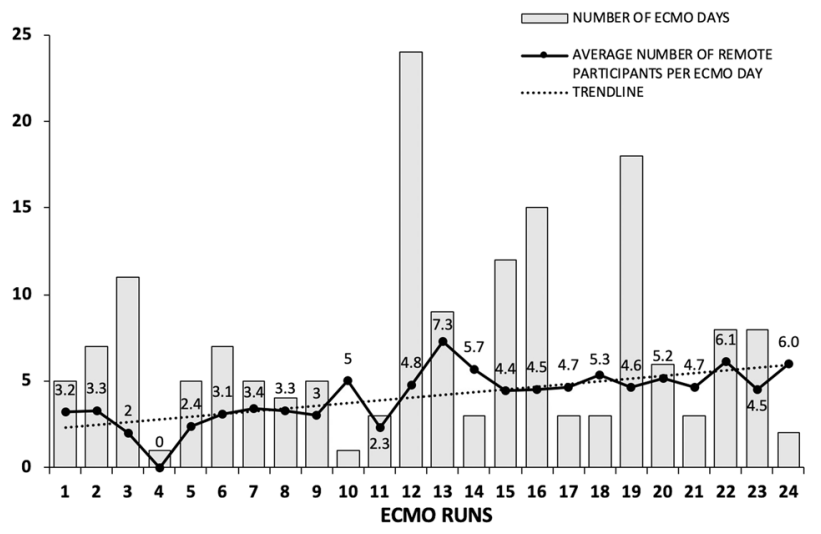

Fig. 2 Average number of remote participants per ECMO run day.

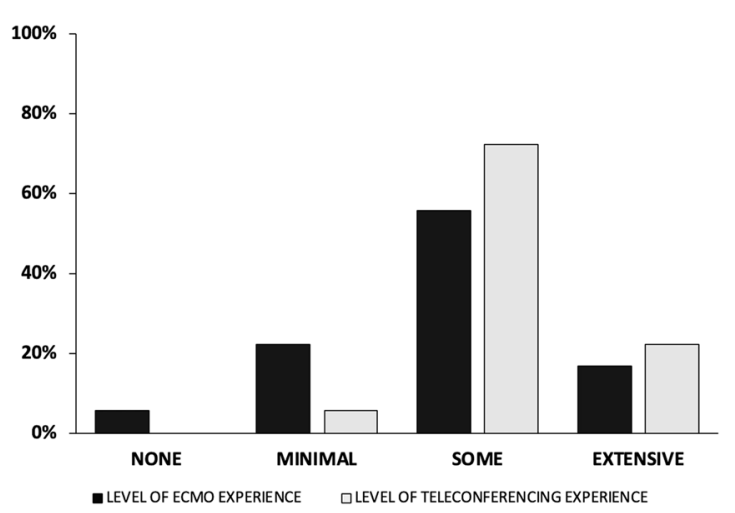

Fig. 3 Participant level of ECMO and telerounding experience.

is shown in Fig. 3. Most participants had at least some experience with both ECMO and teleconferencing. The majority of providers felt comfortable or very comfortable $(12,67 \%)$ with addressing common ECMO clinical management as well as known complications.

Participants were able to join ECMO rounds from home $(13,72 \%)$, from another hospital $(8,44 \%)$, from their office $(6,33 \%)$, and while commuting $(5,28 \%)$. The majority of participants felt that the rounding sheet with a one-line synopsis of the patient $(12,67 \%)$ and the ability to type a question into a chat box and have it answered $(10,56 \%)$ were the most helpful components of telerounding. The feasibility with personnel and equipment, engagement with discussion, audio quality, educational value, value to patient care and continuity, and overall satisfaction of the program were ranked from "very poor" to "very good", with a majority of participants ranking the program "fair" to "very good" (Fig. 4). Nearly all participants felt teleconferencing lowered barriers to attend ECMO rounds $(17,94 \%)$, gave the option to be engaged $(16,89 \%)$, felt their continuity of care for the patients on ECMO was improved $(14,78 \%)$. The top barriers to utilizing ECMO teleconferencing were suboptimal sound environment due to inability to hear conversations well and loud ambient noise levels $(12,66 \%)$ 


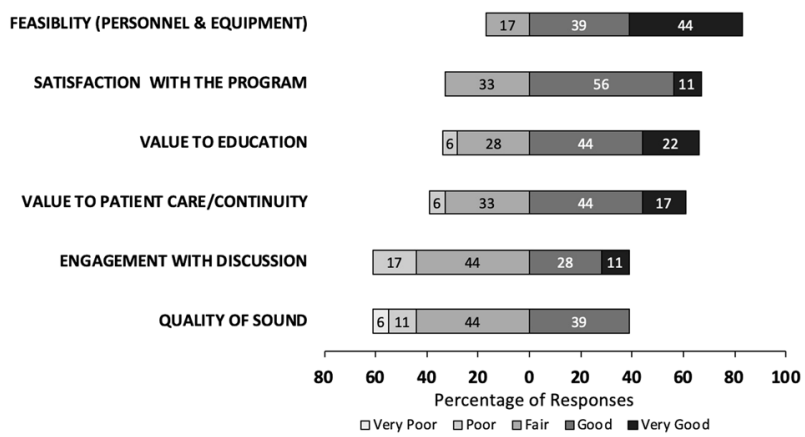

Fig. 4 ECMO telerounding experience.

and the inability to participate more actively in discussion $(6,33 \%)$.

Examples of topics discussed on ECMO rounds included anticoagulation management, fluid and nutrition balance, diuretic use and continuous renal replacement therapy, plasmapheresis, ventilator and lung recruitment strategies, troubleshooting cannula position, surgical timing for repair of congenital diaphragmatic hernia, medication titration of sedation, choice of antibiotics and vasoactive drugs, utility of ancillary radiographic studies such as neuroimaging, abdominal and renal ultrasound, doppler vascular imaging and echocardiography, and timing of trialing off ECMO. Additionally, common ECMO pump related issues such as the circuit cutting-out, arterial and venous limb/cannula thrombosis, bleeding, and oxygenator failure were addressed.

\section{Discussion}

This study looked at the utility of telerounding on ECMO patients in a Level IV NICU to enhance clinical participation, complement in-person ECMO rounds, and ECMO education. We showed that ECMO telerounds was easy to set up and connect to. It increased provider participation in ECMO rounds, allowed for patient continuity, and complemented existing ECMO education at our institution. The main barriers to telerounding were poor audio quality which we worked to improve and the inability to actively participate in the discussion during telerounds. To our knowledge, this is the first study on teleconferencing as a means to lower barriers to provider participation on neonatal ECMO rounds.

We found ECMO telerounds to be feasible in terms of both equipment and personnel participation. The set up using a Bluetooth wireless conference speaker connected to an iPad was easy and quick to complete. NICU providers could connect to telerounds by Zoom app, weblink or calling-in by phone, details of which were readily available in the pre-rounds email. Surveyed NICU providers rated feasibility of equipment set up and connecting to telerounds fair to very good. The Zoom platform has been used for various recent endeavors such as to facilitate focus group discussions among transgender females regarding the acquisition of HIV, to provide collaborative care such as the delivery of cognitive behavioral therapy for the management of youth with post-concussive symptoms, and to deliver quality multi-institutional neonatal education using the "Megaflip" classroom model [16-18]. Zoom's ability to provide synchronized computer mediated communication has the potential for widespread dissemination both in the clinical and educational realms of medicine.

Telerounding increased provider participation and provided continuity of care within our health system. Many physician practices provide clinical coverage to several hospital facilities or clinics and coverage schedules may not always support the continuity of patient care. In our division, neonatologists and advanced practice providers cover nine NICUs and receive neonatal ECMO transports from the Pacific Northwest region, a geographic area that encompasses one-quarter of the U.S. landmass. ECMO telerounds allows the providers within our division who may have cared for the patient prior to transfer to the Level IV unit to participate in ongoing clinical discussions, improving the continuity of care, and decreasing barriers for providers to participate in the care of these critical patients. Nearly all survey respondents agreed that teleconferencing ECMO rounds decreased barriers for participation. This finding is similar to that of the Project Extension for Community Health Outcomes (ECHO) Colorado initiative which focused on developing a statewide network to engage specialists, primary care providers, and other organizations to impart specialty care knowledge and skills for caring for those with complex health conditions in a coordinated statewide approach. The ECHO Colorado initiative utilized the Zoom video communications platform to foster a collaborative education model for healthcare workers in Colorado. On a survey assessing participant engagement, many participants found that the Zoom platform aided in attending sessions due to the flexibility to join from a location of the participant's choice, thus eliminating the geographical barrier to participation [19]. Teleconferencing software has been deployed in many institutions to support physical distancing due to the COVID-19 emergency response and is emerging as an alternative medium for clinical care and education [20, 21]. ECMO telerounding fosters social distancing while maximizing provider participation and the continuity of patient care in the era of COVID-19.

Participants in the study reported that telerounding supported ECMO education for healthcare professionals and learners on geographically distributed campuses. Our neonatal fellows rotate between the level III delivery center and our level IV NICU, so exposure to ECMO care is variable 
during a 3-year fellowship. Given the geographic separation of our units and the infrequency of ECMO runs, telerounding supports clinical participation and ECMO education for our neonatal fellows. The use of teleconferencing in promoting attendance at educational sessions is reflected in other fields such as surgery, in which one study utilized teleconferencing for weekly morbidity and mortality conference with a notable increase in surgical faculty participation [22]. When considering ECMO education, it is imperative to incorporate educational strategies that appeal to adult learning styles, targeting cognitive, technical, and behavioral skills through active learning including discussion, simulation, and real-life patient experiences [23]. Participation in teleconferencing for medical education will likely continue to increase as millennial providers prefer educational instruction that is convenient, technologically enhanced, interactive, and relevant to their field [24].

We identified several barriers to ECMO telerounding. The biggest barrier to participation was poor audio quality due to loud ambient sounds such as patient alarms and the inability to hear conversations due to distance from the conference speaker. Care should be taken to configure the speaker closely to the person speaking in the unit to try to minimize audio interference that can take away from the learning environment [25]. One method found to improve sound was to place the Bluetooth conference speaker on a rolling cart in the middle of the rounding team, allowing it to be easy moved closer to the team member speaking. Additionally, by using Zoom's computer audio, or Voice over Internet Protocol on one's computer or iPad, background ambient noise may be more easily suppressed, thus enhancing the audio quality of the teleconferencing experience [26]. The second most frequent barrier to participation was the inability to actively participate in the discussion during telerounds. All ECMO related questions were asked by telerounding participants via the HIPAA compliant teleconferencing software chat box and were answered by the ECMO attending physician. Every effort was made to address these questions in a timely fashion, and actively during the discussion. Adding video connection during telerounds may further enhance participants feelings of active engagement by allowing remote participants to ask questions face-to-face with the medical team [19].

There are several limitations to our study. First, this study was conducted in a single center Level IV NICU with a division that is accustomed to utilizing telecommunication platforms for meetings and consults. As such, the feasibility and success of teleconferencing ECMO rounds may be difficult to generalize to other NICUs. Second, the survey response rate from participants was $62 \%$, with relatively few fellows and advanced practice providers responding which may skew the data regarding ECMO experience and comfort level. Additionally, the survey was subject to recall bias as providers may have participated remotely months before it was administered. Furthermore, a provider's perception and opinions may have changed since the survey was administered owing to the advancement of the program. Third, this study was conducted to determine the feasibility of teleconferencing for ECMO and as such data collection was tailored to important measures of use and usability without the ability to evaluate its effect on clinical management or educational outcomes. Additionally, provider ECMO knowledge was based off of a self-reported perception survey which focuses on the provider's perception of their skills and knowledge. In order to fully assess the educational impact of ECMO teleconferencing, future studies should consider conducting baseline and longitudinal assessments on ECMO knowledge and practical skills.

\section{Conclusion}

ECMO telerounds is feasible to conduct using the teleconferencing platform, Zoom, and is well-received by neonatal providers. ECMO telerounds can improve provider participation in ECMO cases, provide patient continuity, and complement existing in-person ECMO rounds while fostering a more unified model for ECMO education. Efforts should be made to optimize audio quality during telerounding and consider adding video to improve engagement in telerounds. Future studies are needed to assess the educational impact of ECMO telerounding for providers.

Author contributions BB contributed to data analysis, drafted initial paper, and reviewed and revised the paper. RU, MG contributed to study design, carried out initial analyses, interpretation of data, and reviewed and revised the paper. SH coordinated and supervised data acquisition, reviewed, and revised the paper. RD, TS, and KS interpreted the analyses of the study and critically reviewed the paper for important intellectual content. ZB conceptualized and designed the study, coordinated and supervised data collection, carried out initial analysis and interpretation of analyses, reviewed, and revised the paper. All authors approved the final paper as submitted and agree to be accountable for all aspects of the work.

\section{Compliance with ethical standards}

Conflict of interest The authors declare that they have no conflict of interest.

Publisher's note Springer Nature remains neutral with regard to jurisdictional claims in published maps and institutional affiliations.

\section{References}

1. Bhatt P, Lekshminarayanan A, Donda K, Dapaah-Siakwan F, Patel A, Parat S, et al. National trends in neonatal extracorporeal 
membrane oxygenation in the United States. J Perinatol. 2018;38:1106-13.

2. Mahmood B, Newton D, Pallotto EK. Current trends in neonatal ECMO. Semin Perinatol. 2018;42:80-8.

3. ELSO International Registry Report. https://www.elso.org/Portals/ 0/Files/Reports/2017/International\%20Summary\%20January\% 202017.pdf. Accessed 8 May 2020.

4. Gonzalez DO, Sebastião YV, Cooper JN, Minneci PC, Deans KJ. Pediatric extracorporeal membrane oxygenation mortality is related to extracorporeal membrane oxygenation volume in US hospitals. J Surg Res. 2019;236:159-65.

5. ELSO Guidelines for Training and Continuing Education of ECMO Specialists. https://www.elso.org/Portals/0/IGD/Archive/ FileManager/97000963d6cusersshyerdocumentselsoguidelinesfor trainingandcontinuingeducationofecmospecialists.pdf. Accessed 25 Oct 2019.

6. Muratore S, Beilman G, John R, Brunsvold M. Extracorporeal membrane oxygenation credentialing: where do we stand? Am J Surg. 2015;210:655-60.

7. Patel J, Posencheg M, Ades A. Proficiency and retention of neonatal resuscitation skills by pediatric residents. Pediatrics. 2012;130:515-21.

8. Johnston L, Oldenburg G. Simulation for neonatal extracorporeal membrane oxygenation teams. Semin Perinatol. 2016;40:421-9.

9. Whitehall J, Bliganault I, French C, Carson V, Patole S. Telemedicine in neonatology: lessons from North Queensland. Aust J Rural Health. 1998;6:140-3.

10. Fang JL, Collura CA, Johnson RV, Asay GF, Carey WA, Derleth DP, et al. Emergency video telemedicine consultation for newborn resuscitations: the Mayo clinic experience. Mayo Clin Proc. 2016;91:1735-43.

11. Garingo A, Friedlich P, Chavez T, Tesoriero L, Patil S, Jackson P, et al. 'Tele-rounding' with a remotely controlled mobile robot in the neonatal intensive care unit. $\mathrm{J}$ Telemed Telecare. 2016;22:132-8.

12. Makkar A, McCoy M, Hallford G, Escobedo M, Szyld E. A hybrid form of telemedicine: a unique way to extend intensive care service to neonates in medically underserved areas. Telemed E Health. 2018;24:717-21.

13. Fang JL, Carey WA, Lang TR, Lohse CM, Colby CE. Realtime video communication improves provider performance in a simulated neonatal resuscitation. Resuscitation. 2014; 85:1518-22.

14. Hoffman AM, Lapcharoensap W, Huynh T, Lund K. Historical perspectives: telemedicine in neonatology. NeoReviews. 2019;20: e113-23.
15. Lopez-Magallon AJ, Saenz L, Lara Gutierrez J, Florez CX, Althouse AD, Sharma MS, et al. Telemedicine in pediatric critical care: a retrospective study in an international extracorporeal membrane oxygenation program. Telemed E Health. 2017;24:489-96.

16. Wirtz AL, Cooney EE, Chaudhry A, Reisner SL. American Cohort To Study HIVAATW. Computer-mediated communication to facilitate synchronous online focus group discussions: feasibility study for qualitative HIV research among transgender women across the United States. J Med Internet Res. 2019;21: e12569.

17. McCarty CA, Zatzick D, Hoopes T, Payne K, Parrish R, Rivara FP. Collaborative care model for treatment of persistent symptoms after concussion among youth (CARE4PCS-II): study protocol for a randomized, controlled trial. Trials. 2019;20:567.

18. Beer L, Gray M, Carbajal MM, French H, Vasquez M, Bauserman $\mathrm{M}$, et al. "Megaflip", a novel approach to national collaboration for flipped classroom education. Acad Pediatr. 2020. https://doi. org/10.1016/j.acap.2020.05.027.

19. Shimasaki S, Bishop E, Guthrie M, Thomas JFF. Strengthening the health workforce through the ECHO stages of participation: participants' perspectives on key facilitators and barriers. J Med Educ Curric Dev. 2019;6:2382120518820922.

20. Grange ES, Neil EJ, Stoffel M, Singh AP, Tseng E, RescoSummers K, et al. Responding to COVID-19: the UW medicine information technology services experience. Appl Clin Inf. 2020;11:265-75.

21. Chick RC, Clifton GT, Peace KM, Propper BW, Hale DF, Alseidi AA, et al. Using technology to maintain the education of residents during the COVID-19 pandemic. J Surg Educ. 2020. https://doi. org/10.1016/j.jsurg.2020.03.018.

22. Falcone JL, Watson AR. Surgical morbidity and mortality conference using teleconferencing allows for increased faculty participation and moderation from satellite campuses and saves costs. J Surg Educ. 2012;69:58-62.

23. Johnston L, Williams SB, Ades A. Education for ECMO providers: using education science to bridge the gap between clinical and educational expertise. Semin Perinatol. 2018;42:138-46.

24. Hopkins L, Hampton BS, Abbott JF, Buery-Joyner SD, Craig LB, Dalrymple JL, et al. To the point: medical education, technology, and the millennial learner. Am J Obstet Gynecol. 2018;218:188-92.

25. Lamba P. Teleconferencing in medical education: a useful tool. Australas Med J. 2011;4:442-7.

26. Have no fear: zoom computer audio is here! zoom blog. 2018. https://blog.zoom.us/wordpress/2018/03/19/no-fear-zoomcomputer-audio/. Accessed 27 Apr 2020. 